NASA/CR-2003-212098

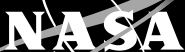

Noise Generation by Fans With Supersonic Tip Speeds

Stewart Glegg

Florida Atlantic University, Boca Raton, Florida 
Since its founding, NASA has been dedicated to the advancement of aeronautics and space science. The NASA Scientific and Technical Information (STI) Program Office plays a key part in helping NASA maintain this important role.

The NASA STI Program Office is operated by Langley Research Center, the Lead Center for NASA's scientific and technical information. The NASA STI Program Office provides access to the NASA STI Database, the largest collection of aeronautical and space science STI in the world. The Program Office is also NASA's institutional mechanism for disseminating the results of its research and development activities. These results are published by NASA in the NASA STI Report Series, which includes the following report types:

- $\quad$ TECHNICAL PUBLICATION. Reports of completed research or a major significant phase of research that present the results of NASA programs and include extensive data or theoretical analysis. Includes compilations of significant scientific and technical data and information deemed to be of continuing reference value. NASA's counterpart of peerreviewed formal professional papers but has less stringent limitations on manuscript length and extent of graphic presentations.

- TECHNICAL MEMORANDUM. Scientific and technical findings that are preliminary or of specialized interest, e.g., quick release reports, working papers, and bibliographies that contain minimal annotation. Does not contain extensive analysis.

- CONTRACTOR REPORT. Scientific and technical findings by NASA-sponsored contractors and grantees.
- CONFERENCE PUBLICATION. Collected papers from scientific and technical conferences, symposia, seminars, or other meetings sponsored or cosponsored by NASA.

- SPECIAL PUBLICATION. Scientific, technical, or historical information from NASA programs, projects, and missions, often concerned with subjects having substantial public interest.

- TECHNICAL TRANSLATION. Englishlanguage translations of foreign scientific and technical material pertinent to NASA's mission.

Specialized services that complement the STI Program Office's diverse offerings include creating custom thesauri, building customized databases, organizing and publishing research results ... even providing videos.

For more information about the NASA STI Program Office, see the following:

- Access the NASA STI Program Home Page at http://www.sti.nasa.gov

- E-mail your question via the Internet to help@sti.nasa.gov

- Fax your question to the NASA Access Help Desk at 301-621-0134

- Telephone the NASA Access Help Desk at 301-621-0390

- Write to:

NASA Access Help Desk

NASA Center for AeroSpace Information 7121 Standard Drive

Hanover, MD 21076 
NASA/CR-2003-212098

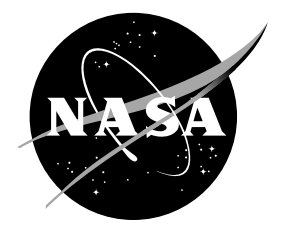

\section{Noise Generation by Fans With Supersonic Tip Speeds}

Stewart Glegg

Florida Atlantic University, Boca Raton, Florida

Prepared under Grant NAG3-2702

National Aeronautics and

Space Administration

Glenn Research Center 
This report contains preliminary findings, subject to revision as analysis proceeds.

The Propulsion and Power Program at NASA Glenn Research Center sponsored this work.

National Technical Information Service 5285 Port Royal Road

NASA Center for Aerospace Information

Available from
Springfield, VA 22100 


\title{
Noise Generation by Fans With Supersonic Tip Speeds
}

\author{
Stewart Glegg \\ Center for Acoustic and Vibration \\ Florida Atlantic University \\ Boca Raton, Florida 33431
}

\section{Introduction}

Fan noise continues to be a significant issue for commercial aircraft engines and there still exists a requirement for improved understanding of the fundamental issues associated with fan noise source mechanisms. At the present time, most of the prediction methods identify the dominant acoustic sources to be associated with the stator vanes or blade trailing edges which are downstream of the fan face (Morin 1999). However recent studies (Hanson 2002) have shown that acoustic waves are significantly attenuated as they propagate upstream through a rotor, and if the appropriate corrections are applied, sound radiation from the engine inlet is significantly underpredicted. The prediction models can only be applied to fans with subsonic tip speeds. In contrast, most aircraft engines have fan tip speeds which are transonic and this implies an even higher attenuation for upstream propagating acoustic waves. Consequently understanding how sound propagates upstream through the fan is an important, and not well understood phenomena. The objective of this study is to provide improved insight into the upstream propagation effects through a rotor which are relevant to full scale engines.

The focus of this study is on broadband fan noise generated by boundary layer turbulence interacting with the trailing edges of the fan blades. If this source mechanism is important upstream of the fan, the sound must propagate upstream through a transonic non uniform flow which includes large gradients and non linearities. Developing acoustic propagation models in this type of flow is challenging and currently limited to low frequency applications, where the frequency is of the same order as the blade passing frequency of the fan. For trailing edge noise, much higher frequencies are relevant and so a suitable approach needs to be developed, which is not limited by an unacceptably large computational effort. In this study we are in the process of developing a computational method which applies for the high frequencies of interest, and allows for any type of flow field associated with the fan. In this progress report the approach to be used and the basic equations will be presented. Some initial results will be given, but these are preliminary and need further verification.

\section{Approach}

As was described in the proposal for this study, the most suitable formulation for the study of trailing edge noise is the wave equation developed by Howe, which uses the stagnation enthalpy $B$ as the dependent variable and specifies a dipole source term determined by the local vorticity $\omega$, the temperature $T$ and entropy $S$. The equation is given as

$$
\frac{D}{D t}\left(\frac{1}{c^{2}} \frac{D B}{D t}\right)-\frac{1}{\rho} \nabla(\rho \nabla B)=\nabla(\boldsymbol{\omega} \times \mathbf{u}-T \nabla S)
$$


In this equation the density $\rho$ and speed of sound $c$ vary spatially and the total time derivative is linearized about the mean flow so that

$$
\frac{D}{D t} \equiv \frac{\partial}{\partial t}+\mathbf{U} \cdot \nabla
$$

In order to develop a general high frequency theory, we will consider the homogeneous form of this equation and introduce the source terms at the boundaries or the domain. Hence we define a region where a function $f>0$ in which the right hand side of (1) is zero so that

$$
\begin{aligned}
& \frac{D}{D t}\left(\frac{1}{c^{2}} \frac{D B}{D t}\right)-\frac{1}{\rho} \nabla(\rho \nabla B)=0 \quad f>0 \\
& \text { where } \\
& B=B_{q} \quad f=0
\end{aligned}
$$

We will seek a solution in the high frequency limit where $k_{o}=\omega / c_{o}$ becomes very large and $c_{o}$ is the speed of sound in a region of uniform flow far upstream of the region of interest. If we consider a solution in the form

$$
B=B_{o} e^{-i \omega t+i k_{o} q(x, y)}
$$

Substituting (3) into (2) we obtain

$$
\left\{k_{o}^{2}\left[\nabla q . \nabla q-n^{2}(1-\mathbf{M} . \nabla q)^{2}\right]-i k_{o}\left[\nabla^{2} q+\mathbf{M} . \nabla\left(n^{2}(1-\mathbf{M} . \nabla q)\right)+\frac{\nabla \rho . \nabla q}{\rho}\right]\right\} B_{o} e^{-i \omega t+i k_{o} q(x, y)}=0
$$

In the high frequency limit the second term can be ignored so we obtain a single equation for $q$ which gives the non linear equation

$$
\nabla q \cdot \nabla q-n^{2}(1-\mathbf{M} \cdot \nabla q)^{2}=0
$$

However in general both terms in (4) need to be considered giving the equation for $q$ as

$$
\left[\nabla q . \nabla q-n^{2}(1-\mathbf{M} . \nabla q)^{2}\right]-\frac{i}{k_{o}}\left\lceil\nabla^{2} q+\mathbf{M} \cdot \nabla\left(n^{2}(1-\mathbf{M} . \nabla q)\right)+\frac{\nabla \rho . \nabla q}{\rho}\right]=0
$$

\section{Simple Examples}

To understand this equation first consider examples where there is no flow and the mean density is constant so (6) reduces to

$$
\nabla q . \nabla q=n^{2}+\frac{i}{k_{o}} \nabla^{2} q
$$


For cylindrical waves this reduces to

$$
\left(\frac{\partial q}{\partial R}\right)^{2}+\frac{1}{R^{2}}\left(\frac{\partial q}{\partial \theta}\right)^{2}=n^{2}+\frac{i}{k_{o}}\left(\frac{1}{R} \frac{\partial}{\partial R}\left(R \frac{\partial q}{\partial R}\right)+\frac{1}{R^{2}}\left(\frac{\partial^{2} q}{\partial \theta^{2}}\right)\right)
$$

If the boundary condition is independent of $\theta$ and the speed of sound is constant then

$$
\frac{\partial q}{\partial R}= \pm\left[1+\frac{i}{k_{o}}\left(\frac{1}{R} \frac{\partial}{\partial R}\left(R \frac{\partial q}{\partial R}\right)\right)\right]^{1 / 2}
$$

In the high frequency limit $\bullet q / \bullet R \sim 1$ and so to first order in $1 / k_{o}$ we have

$$
\frac{\partial q}{\partial R} \approx\left\lfloor\left[1 \pm \frac{i}{k_{o}}\left(\frac{1}{R} \frac{\partial}{\partial R}(R)\right)\right]^{1 / 2} \approx \pm\left[1 \pm \frac{i}{2 k_{o} R}\right\rceil\right.
$$

and integration yields

$$
q \approx \pm R+\frac{i}{2 k_{o}} \log _{e}\left(k_{o} R\right)
$$

Hence we find as expected that $B=\exp \left( \pm i k_{o} R\right) /\left(k_{o} R\right)^{1 / 2}$ but we note that the effect of cylindrical spreading is determined by the Laplacian in equation (6).

Another example is given by a dipole source which can be modeled by a boundary condition which depends on $\cos \theta$. Then the full form of (8) must be used, but if $n$ is constant we find

$$
R^{2}\left(\frac{\partial q}{\partial R}\right)^{2}-\frac{i}{k_{o}}\left(R \frac{\partial}{\partial R}\left(R \frac{\partial q}{\partial R}\right)\right)-R^{2}=-\left(\frac{\partial q}{\partial \theta}\right)^{2}+\frac{i}{k_{o}} \frac{\partial^{2} q}{\partial \theta^{2}}
$$

The boundary condition implies that $q=f(R)+g(\theta)$ where $g=1 /\left(i k_{o}\right) \log (\cos \theta)$. It follows that

$$
R^{2}\left(\frac{\partial f}{\partial R}\right)^{2}-\frac{i}{k_{o}}\left(R \frac{\partial}{\partial R}\left(R \frac{\partial f}{\partial R}\right)\right)-R^{2}=\frac{1}{k_{o}^{2}}(\tan \theta)^{2}-\frac{1}{k_{o}^{2}}(\sec \theta)^{2}=\frac{-1}{k_{o}^{2}}
$$

and so

$$
\frac{\partial}{\partial R}=\left\lceil 1-\frac{1}{\left(k_{o} R\right)^{2}}+\frac{i}{k_{o} R}\left(\frac{\partial}{\partial R}\left(R \frac{\partial f}{\partial R}\right)\right)^{1 / 2} \approx\left\lfloor 1+\frac{i}{k_{o} R}\right]^{1 / 2}\right.
$$


giving as before the result that

$$
q \approx \pm R+\frac{i}{2 k_{o}} \log _{e}\left(k_{o} R\right)-\frac{i}{k_{o}} \log _{e}(\cos \theta)
$$

\section{Cartesian Coordinates}

The next step in the analysis is to consider numerical solutions in cartesian coordinates. In the absence of a flow we need to solve

$$
\frac{\partial q}{\partial y}=\left[n^{2}-\left(\frac{\partial q}{\partial x}\right)^{2}+\frac{i}{k_{o}}\left(\frac{\partial^{2} q}{\partial x^{2}}+\frac{\partial^{2} q}{\partial y^{2}}\right)\right]^{1 / 2}
$$

When the flow is primarily in the $x$ direction, we can specify equations suitable for numerical integration as

$$
\frac{\partial q}{\partial y}=\left[n^{2}\left(1-M_{x}\left(\frac{\partial q}{\partial x}\right)\right)^{2}-\left(\frac{\partial q}{\partial x}\right)^{2}+\frac{1}{i k_{o}}\left(\left(1-n^{2} M_{x}^{2}\right) \frac{\partial^{2} q}{\partial x^{2}}+\frac{\partial^{2} q}{\partial y^{2}}\right)\right]^{1 / 2}
$$

\section{Extensions to More Complex Environments}

Three dimensional problems are also readily solved by adding the term $(\partial q / \partial z)^{2}$ to equation (5) and specifying the boundary condition on the surface $y=c o n s t a n t$ as a function of $x$ and $z$. For problems in cylindrical coordinates $(r, \theta, z)$ the natural extension of the above approach is to specify the boundary conditions on $r=$ const and consider radial propagation. For ducted flow however it may be more suitable to specify the boundary conditions on a cross sectional surface $z=$ const and propagate waves up or downstream. This is equivalent to marching the solution in the $x$ direction using (6) with boundary conditions specified on $x=$ const.

Trailing edge noise can be calculated using this approach by specifying the appropriate source distribution in the vicinity of the trailing edge of the blade. This source distribution is well known and specified in the literature (Howe, 1978) for a spatially harmonic disturbance in uniform flow. The most convenient form for the trailing edge noise source distribution is given by the Greens function for a source close to a sharp edge in a uniform flow of Mach number $M$, which is given by

$$
\begin{aligned}
& G=\frac{-i}{8 \pi} \sum_{n=0}^{\infty} \varepsilon_{n}\left(H_{n / 2}^{(1)}\left(k_{o} r^{\prime} / \beta\right) J_{n / 2}\left(k_{o} r_{o}{ }^{\prime} / \beta\right) H\left(r^{\prime}-r_{o}{ }^{\prime}\right)\right. \\
&\left.\left.+H_{n / 2}^{(1)}\left(k_{o} r_{o}{ }^{\prime} / \beta\right) J_{n / 2}\left(k_{o} r^{\prime} / \beta\right) H\left(r_{o}{ }^{\prime}-r^{\prime}\right)\right) \cos \left(\frac{n(\theta-\pi)}{2}\right)\right) e^{i k_{o} M\left(x-x_{o}\right) / \beta^{2}}
\end{aligned}
$$

where $r^{\prime}=\left(x^{2} / \beta^{2}+y^{2}\right)^{1 / 2}$ and the subscript $o$ refers to the observer location. Note that $\varepsilon_{n}=1, n=0$ and $\varepsilon_{n}=2, n>0$. Also $\beta^{2}=1-M^{2}$. 


\section{Preliminary Numerical Results}

Figure 1 shows calculations for the source distribution defined by a Gaussian distribution of pressure on the $\mathrm{x}$ axis and illustrates the propagation of a Gaussian beam. This shows that the procedure can be used successfully to propagate sound waves over many wave lengths. Similar examples have also been computed for monopole and dipole source fields. In general the procedure works well until the value of the Laplacian in equation (16) or (17) becomes negative. The correct choice of the branch cut is crucial to the numerical procedure and work continues to fully understand this issue.

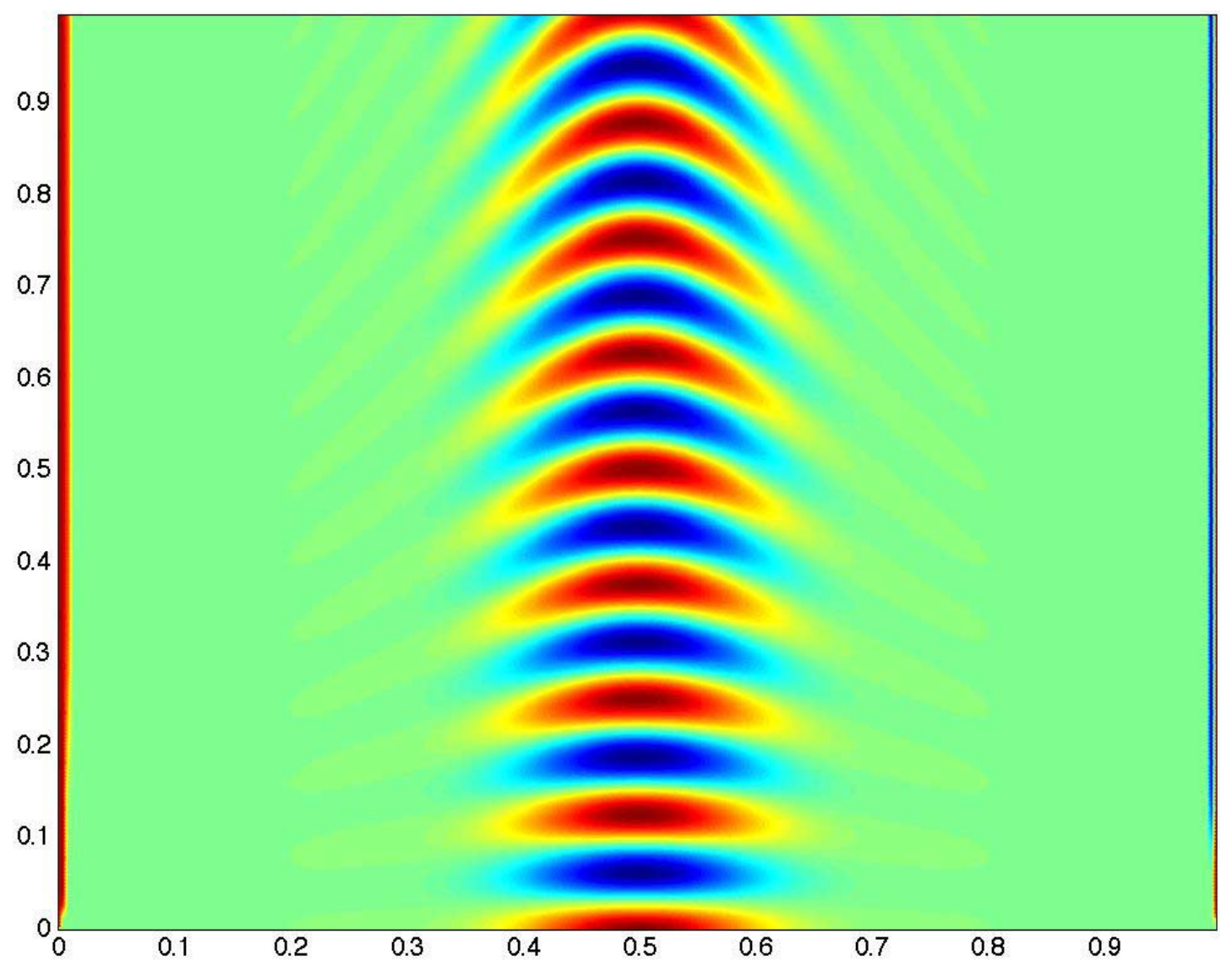

Figure 1: Sound propagation of a Gaussian Beam obtained numerically. 


\section{Computation of the Flow}

To obtain the flow through which the sound will propagate, we need to develop a computational method which can be used specifically for the problem being considered. The computation must provide the flow over a fan blade when the free stream Mach number is between $M=0.7$ and $M=1.4$. To achieve this we will use transonic small disturbance theory which is based on the following assumptions:

1. The airfoil is thin, with a small camber and a small angle of attack

2. The horizontal and vertical components of the velocity field are not very different from their free stream value

The transonic small disturbance equation which will be solved is:

$$
\left\lfloor 1-M_{\infty}^{2}-(\gamma+1) M_{\infty}^{2} \frac{\varphi_{x}}{V_{\infty}}\right\rfloor \varphi_{x x}+\varphi_{y y}=0
$$

where $\varphi_{x x}$ denotes the double derivative of the disturbance velocity potential and $M_{\infty}$ represents the free stream Mach number.

The solution of this nonlinear equation is subject to boundary conditions at the solid boundary of the airfoil, along a cut downstream of the airfoil trailing edge, and at the far field boundaries. On the airfoil, the disturbance velocity potential must satisfy the following condition:

$$
\varphi_{y}=V_{\infty}\left(1+\frac{\varphi_{x}}{V_{\infty}}\right) \cdot \frac{d Y}{d x}
$$

For small disturbance, we can neglect the term $\frac{\varphi_{x}}{V_{\infty}}$ and use the simpler condition:

$$
\varphi_{y}=V_{\infty} \frac{d Y}{d x}
$$

If the airfoil is producing lift, the circulation around the body will not be zero. Far field boundary conditions are also important and for the subsonic free stream case, we use the analytical result:

$$
\varphi=\frac{\Gamma}{2 \pi} \arctan \left(\sqrt{1-M_{\infty}^{2}} \frac{y}{x}\right)
$$

For the supersonic free stream case, the lateral boundaries are computed with the method of characteristics, the disturbance velocity potential at the upstream boundary is set to zero (no perturbation can radiate upstream), the downstream boundary is computed as any other point in the flow. 
To limit the computation time and still obtain good accuracy, we use a stretched grid to define the computational domain. The mesh is fine where the velocity potential gradient is important (near the airfoil) and becomes larger as we get further from the body. This allows having accurate results with a matrix $100 * 120$ instead of a matrix $1380 * 400$ (this divides the computation time by 46$)$.

This approach has been implemented and an example of the computation of with a free stream of Mach number $\mathrm{M}=0.99$ over a double-cosine-shape airfoil is shown in Figure 2.

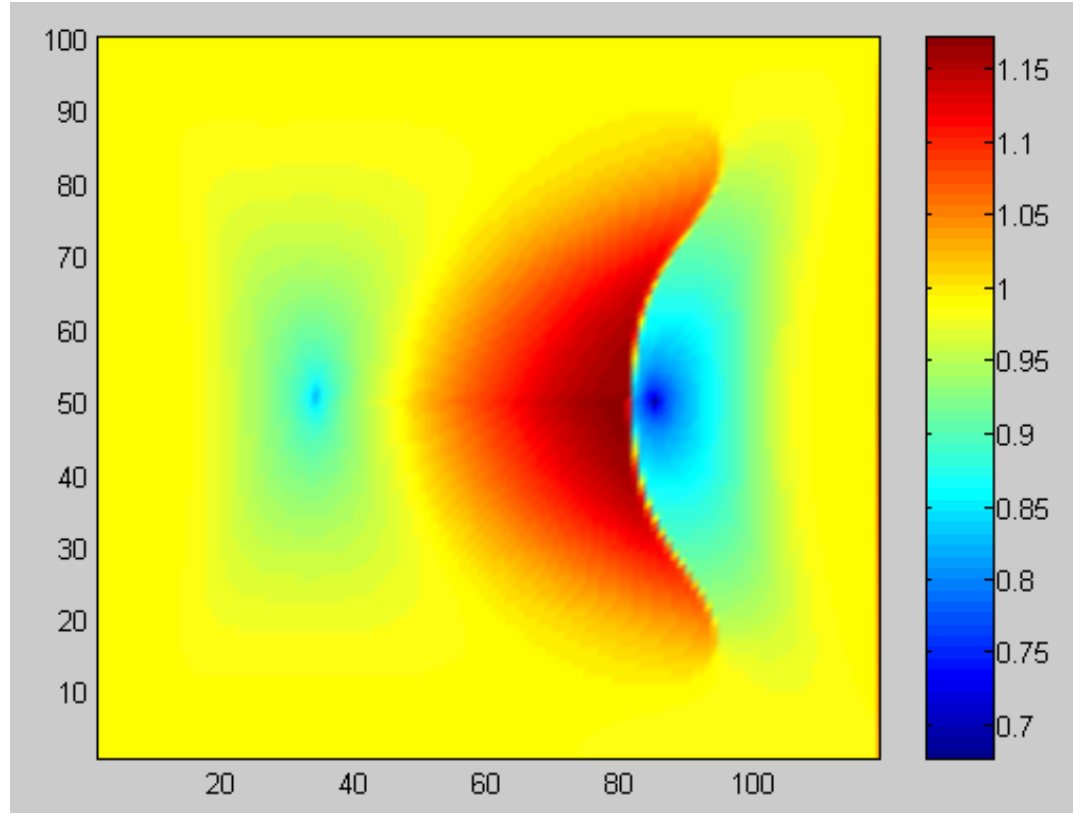

Figure 2: Computation of the flow with a free stream of Mach number $\mathrm{M}=0.99$ over a double-cosine-shape airfoil.

\section{Future Work}

The next steps in this study are

1) Introduce the trailing edge noise source distribution to the acoustic computations, and study the effect of flow on the radiated sound.

2) Introduce the flow computed by transonic small disturbance theory to the acoustic computations and study the effect of more realistic shocks on the acoustic computation.

3) Investigate the effects of shocks on trailing edge noise in two dimensional flow.

4) Introduce a cascade of blades and repeat the above study.

5) Extend the work to three dimensional flows in circular ducts. 


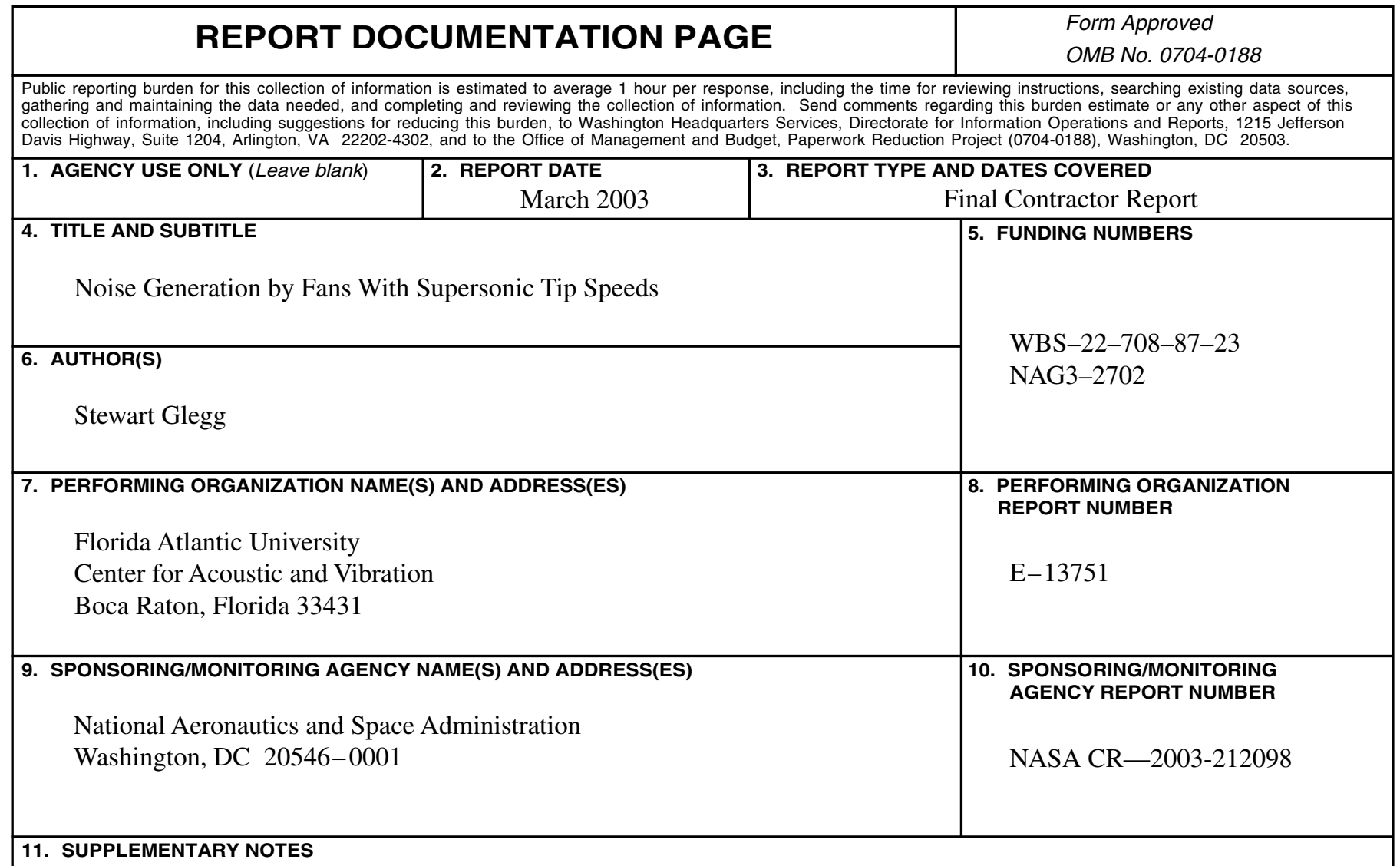

Project Manager, Edmane Envia, Structures and Acoustics Division, NASA Glenn Research Center, organization code 5940, 216-433-8956.

12a. DISTRIBUTION/AVAILABILITY STATEMENT 12b. DISTRIBUTION CODE

Unclassified - Unlimited

Subject Category: 02

Distribution: Nonstandard

Available electronically at http://gltrs.grc.nasa.gov

This publication is available from the NASA Center for AeroSpace Information, 301-621-0390.

13. ABSTRACT (Maximum 200 words)

The focus of this study is on broadband fan noise generated by boundary layer turbulence interacting with the trailing edges of the fan blades. If this source mechanism is important upstream of the fan, the sound must propagate upstream through a transonic non uniform flow which includes large gradients and non linearities. Developing acoustic propagation models in this type of flow is challenging and currently limited to low frequency applications, where the frequency is of the same order as the blade passing frequency of the fan. For trailing edge noise, much higher frequencies are relevant and so a suitable approach needs to be developed, which is not limited by an unacceptably large computational effort. In this study we are in the process of developing a computational method which applies for the high frequencies of interest, and allows for any type of flow field associated with the fan. In this progress report the approach to be used and the basic equations will be presented. Some initial results will be given, but these are preliminary and need further verification.

14. SUBJECT TERMS

\begin{tabular}{|l|l|} 
& $\begin{array}{c}15 . \text { NUMBER OF PAGES } \\
13\end{array}$ \\
\cline { 2 - 2 } & 16. PRICE CODE \\
$\begin{array}{l}\text { 19. SECURITY CLASSIFICATION } \\
\text { OF ABSTRACT } \\
\text { Unclassified }\end{array}$ & 20. LIMITATION OF ABSTRACT \\
\hline
\end{tabular}

NSN 7540-01-280-5500

Standard Form 298 (Rev. 2-89)

Prescribed by ANSI Std. Z39-18 298-102 